\title{
Exploring the Role of Platelet Derived Concentrates in Accelerated Orthodontics - A Narrative Review
}

\section{Gayatri Ganesh* and Tulika Tripathi}

Department of Orthodontics and Dentofacial Orthopaedics, Maulana Azad Institute of Dental Sciences, Delhi University, India

*Corresponding Author: Gayatri Ganesh, Department of Orthodontics and Dentofacial Orthopaedics, Maulana Azad Institute of Dental Sciences, Delhi University, India.
Received: September 05, 2020

Published: September 30, 2020

(C) All rights are reserved by Gayatri Ganesh and Tulika Tripathi.

\section{Abstract}

Devising new approaches to hasten the regenerative process, thereby, waning the duration of wound healing persists to be one of prime goals of clinical research. Platelets play a crucial role in these regenerative procedures, as they act as reservoirs of cytokines and growth factors. A significant component of the orthodontic tooth movement encompasses a similar process as it essentially involves bone remodeling comprising of alternate cycles of bone resorption and deposition. The past decades have witnessed various attempts to accelerate the rate of orthodontic movement by versatile techniques leading to a decrease in the treatment time, a highly desired outcome by the patient as well as the orthodontist. The following review attempts to summarize existing literature concerning the application of platelet derived concentrates in dentistry and their potential role in orthodontics. The most commonly used platelet derived concentrates i.e Platelet Rich Plasma (PRP), Plasma rich in growth factors (PRGF) and the Platelet rich Fibrin (PRF) have been described and compared to aid in future opportunities of clinical research.

Keywords: Platelet-rich Plasma; Platelet-rich Fibrin; Orthodontics; Regenerative Medicine

\section{Introduction/Description}

Long treatment duration persists to be one the prime concerns perturbing the patient before treatment commencement. More often than not, the long treatment duration associated becomes a deterrent discouraging the patient from undergoing treatment. Consequentially, orthodontic tooth movement and the associated biological reaction persist to be one the prime research domains.

Orthodontic tooth movement involves modeling-remodeling of the alveolar bone caused by a series of inflammatory mediators released on the application of an external force [1-3]. The process of remodeling is essentially a healing process initiated by the formation of a blood clot, followed by a proliferative stage comprising of new epithelium, blood vessel and granulation tissue formation ultimately leading to deposition of collagen and bone [4]. The clot formation involves platelet aggregation and adherence favoring the formation of thrombin and fibrin.
Many surgical and non-surgical approaches have been carried out over the years by researchers to enhance the orthodontic tooth movement while preserving the bone physiology. The surgical category is based on the principle of the Regional Acceleratory Phenomenon described by Frost [5], where surgical irritation of the bone releases an inflammatory cascade leading to osteoclastogenesis. Subsequently, alveolar bone resorption ensues and results in a decrease in the thickness and weight of the alveolar bone, thus, producing accelerated tooth movement [6]. A majority of the surgical procedures such as alveolar decortication, distraction of the periodontal ligament or the dento-alveolus [7] follow this principle and produce an insult to the bony tissue to accelerate tooth movement. However, these interventions were deemed to be traumatic and thus, less invasive and non invasive procedures, thus, such as injections of prostaglandins $[8,9]$, osteocalcin $[9,10]$, active form of vitamin D [8,10], resonance vibration [11], photobiomodulation including low level laser therapy $[12,13]$, LED and ultrasound 
waves [14] were introduced. Though some of the pharmacological substances did stimulate the rate of tooth movement, one could not overlook the undesirable side effects of local pain and discomfort and during a shorter duration of action [6].

The concepts of regenerative surgery where surgical annihilation techniques are often combined with regenerative methods to attain ideal healing have been widely used in various aspects of dentistry such as intrabony defects, gingival recession, sinus lifting and alveolar filling post extraction $[15,16]$. The bioactive properties of platelets in the healing process have enabled them to be used as a therapeutic and non-invasive adjunct for these various processes. Thus, the application of autologous platelet concentrates in the field of orthodontics may prove to be promising and thus warrants the need for extensive research.

\section{Platelet concentrates and their evolution}

Platelet concentrates are products derived from the centrifugation of blood concentrating platelets, leukocytes and fibrin to convert them into a clinically useful form. The efficiency of the concentrate depends on the platelet concentration, number and type of leukocytes in the fibrin membrane and the subsequent release of bioactive molecules from the site of the clot triggering the regenerative process [17]. The bioactive molecules released include growth factors such as platelet-derived growth factor (PDGF), epidermal growth factor (EGF), vascular endothelial growth factor (VEGF), insulin-like growth factor (IGF-I), hepatocyte growth factor (HGF), transforming growth factor- $\beta$ (TGF- $\beta$ ) and cytokines such as interleukin-1 (IL-1), interleukin-4 (IL-4), interleukin-6 (IL6), interleukin-10 (IL-10) and chemotactic molecules such as exotoxin and chemokine ligand-5 (CCL- 5) [18]. The initial protocols involved the formation of first-generation platelet concentrates i.e. the platelet-rich plasma (PRP) and plasma rich in growth factors (PRGF). The expansion in knowledge about the biological features of the concentrates led to the development of the second-generation concentrates i.e. leukocyte-platelet-rich fibrin (L-PRF/PRF). The evolution is based on the principle of addition of no anticoagulants and no manipulation of the blood sample to obtain the concentrates [19]. Further advances in the field of platelet concentrates include the development of CGF (Concentrated Growth Factors), A-PRF (Advanced PRF) and i-PRF (injectable form of PRF). Concentrated Growth Factors (CGF) were introduced by Sacco [20] in 2006 where the preparation of the concentrates is similar to PRF but at a different speed. This allowed the separation and forma- tion of a fibrin matrix which was much larger, denser and richer in growth factors. Choukroun [21] further modified the L-PRF and introduced a concentrate called the A-PRF in an attempt to incorporate monocytes in the PRF and produce faster soft tissue growth, more growth factors and cytokines release. An injectable form of PRF called the i-PRF was described by Mourão., et al. [22] in 2015, wherein he obtained an orange color fluid instead of the matrix by a shorter centrifugation of blood. This fluid could either be mixed with the graft or injected separately.

However, as these advances are fairly new, no conclusive long term or controlled trials in humans or animals have been carried out to prove their efficiency over the conventional PRP and PRF. Thus, researchers have cautioned against the use of these products till further conclusive results have been attained.

\section{Platelet rich plasma}

Platelet rich plasma or PRP was introduced in dentistry by Robert Marx [23] to enhance the radiographic maturation rate of bone graft in mandibular reconstructive procedures. PRP is an autologous concentration of human platelets in a small volume of plasma comprising of a high concentration of platelets with fundamental growth factors actively secreted by them during wound healing [24]. Platelet counts in PRP are approximately $1,000,000 / \mu 1 \mathrm{com}-$ pared the normal range of $150,000 / \mu 1$ to $350,000 / \mu 1$ [25].

\section{Composition [26]}

Platelet rich plasma (PRP) comprises of:

High concentration of platelets which release the following growth factors:

- $\quad$ Three isomers of platelet-derived GF (PDGFaa, PDGFbb, and PDGFab)

- $\quad$ Transforming GFs-b (TGFb1 and TGFb2),

- $\quad$ Vascular endothelial GF (VEGF)

- $\quad$ Epidermal GF (EGF).

Small volume of plasma containing

- $\quad$ Cytokines, Interleukins and Tumour Necrotic Factor

- $\quad$ Cell adhesion proteins - Fibrin, Fibronectin and Vitronectrin

- $\quad$ Proteases and anti proteases. 
Method of preparation [27]

According to a study by Raja and Naidu [27], PRP can be prepared by two techniques differing in their technical aspects.

\section{General purpose separators}

General-purpose cell separators such as ELMD-500 (Medtronic Electromedic, Auto Transfusion System, Parker, CO, USA) requires a large quantity of blood (approximately $450 \mathrm{ml}$ ) and are usually operated in an hospital setting.

\section{Platelet-concentrating cell separators}

These separators require a smaller quantity of blood (10 - 50 $\mathrm{mL}$ ) to produce PRP and thus are more commonly used in dentistry e.g. Harvest SmartPrep Platelet Concentrate System (HSPCS; Harvest Technologies, Plymouth, MA, USA) and the 3i Platelet Concentrate Collection System (3i PCCS; 3i Implant Innovations, Palm Beach Gardens, FL, USA).

Robert Max originally produced PRP with a double centrifugation technique as a single spin would not produce a true PRP but a mixture of platelet poor plasma and platelet rich plasma with very low platelet counts [24]. The processing of platelet rich plasma essentially differs in the type of anticoagulant and duration of centrifugation in the various platelet-concentrating systems.

PRP is usually obtained in an injectable form but the addition of bovine thrombin and calcium chloride just before application converts it into a gel form. Calcium chloride is added to nullify the effect of the citrate anticoagulant used earlier and the thrombin aids in activation of fibrinogen required to form fibrin [28]. However, in the field of orthodontics, an injectable form without the use of calcium chloride and thrombin has been advocated to have a longer lasting effect of the product [9] (Figure 1).

\section{Applications}

\section{PRP and Accelerated orthodontics}

A study by Graziani., et al. [29] has stated that the role of PRP in bone metabolism is highly dependent of the concentration of the platelets in the product. Rashid., et al. [30] and Güleç., et al. [31] in their animal studies have found significant results in the role of PRP in accelerating the rate of orthodontic tooth movement. However, there still exists a huge lacuna as there is a paucity of any conclusive human studies concerning the same.
45-60 ml of venous blood is drawn with an anticoagulant (Citrate Phosphate Dextrose Adenine) to avoid platelet activation and degranulation. The amount of blood drawn may vary according to the manufacturer's instructions

\section{$\sqrt{4}$}

The first spin (called the hard spin) of centrifugation divides the blood into 3 layers - the bottom most RBC layer ( $55 \%$ of total volume), an intermediate layer i.e buffy coat ( $5 \%$ of total volume) and the topmost acellular plasma layer called PPP ( $40 \%$ of total volume)

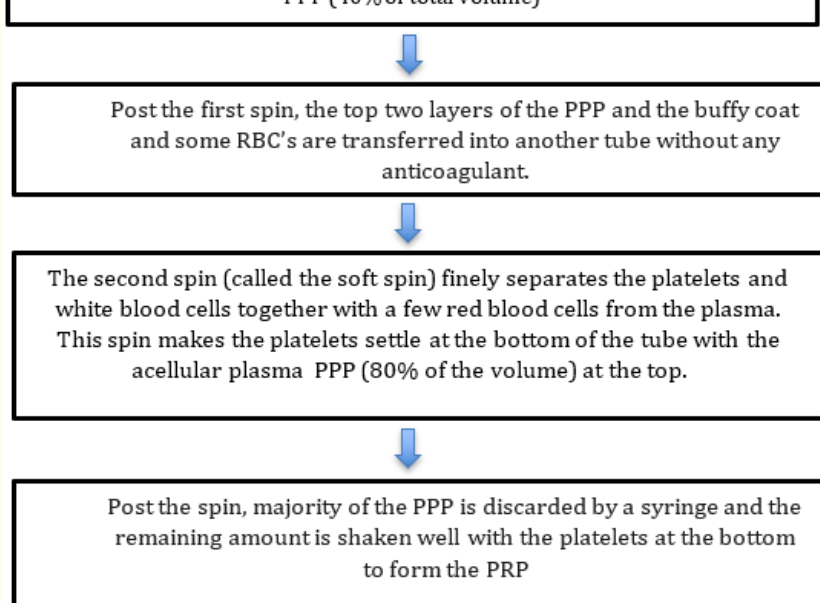

Figure 1: Steps in preparation of PRP.

PRP for alveolar bone grafting in cleft patients

The role of PRP in cleft patients is based on the release of the growth factors by the platelets to aid in bone graft maturation. In a study by Gupta., et al. [32], secondary alveolar bone grafting with the use of PRP showed significantly higher bone density up to 6-months post-surgery. Over the next few years, various studies ${ }^{33,34}$ have elaborated the role of PRP used with an autologous bone graft or other bone substitutes and have demonstrated the positive effect of PRP on the soft tissue by increasing the cell tropism and accelerating the healing process.

\section{Drawbacks of PRP}

The PRP concentrate does not promote bacterial proliferation, as it is similar to a natural clotting process. The growth factors released by the platelets in PRP are only trans-membranous and not mutagenic, thus they simulate the natural healing process without causing any tumor [2].

\section{Plasma rich in growth factors}

To overcome the drawback associated with PRP such as the low handling efficiency or the addition of thrombin [35], PRGF (Plasma 
rich in growth factors) was developed by Anitua E [36] by introducing a modification in the preparation protocol. The thrombin was replaced with calcium for clotting. Anitua E [36] describes PRGF as an autologous platelet enriched plasma without leukocytes. PRGF contains platelet and plasma growth factors along with plasma proteins such as fibrin, vitronectin and fibronectin involved in the wound healing process.

\section{Composition [20]}

The composition of PRGF is essentially similar to PRP i.e.

\section{Growth factors such as:}

- Platelet-derived growth factors

- Transforming GFs-b (TGFb1 and TGFb2)

- Vascular endothelial GF (VEGF)

- Epidermal GF (EGF)

- Insulin like growth factor (IGF-1).

Plasma containing

- Cytokines, Interleukins and Tumor Necrotic Factor

- $\quad$ Cell adhesion proteins - Fibrin, Fibronectin and Vitronectin

- $\quad$ Proteases and anti proteases.

\section{Method of preparation [33]}

The preparation of PRGF also requires a small volume of blood, which is adapted to each case ranging from $5-80 \mathrm{~cm}^{3}$. Sodium citrate and calcium chloride are added as anticoagulants (Figure 2).

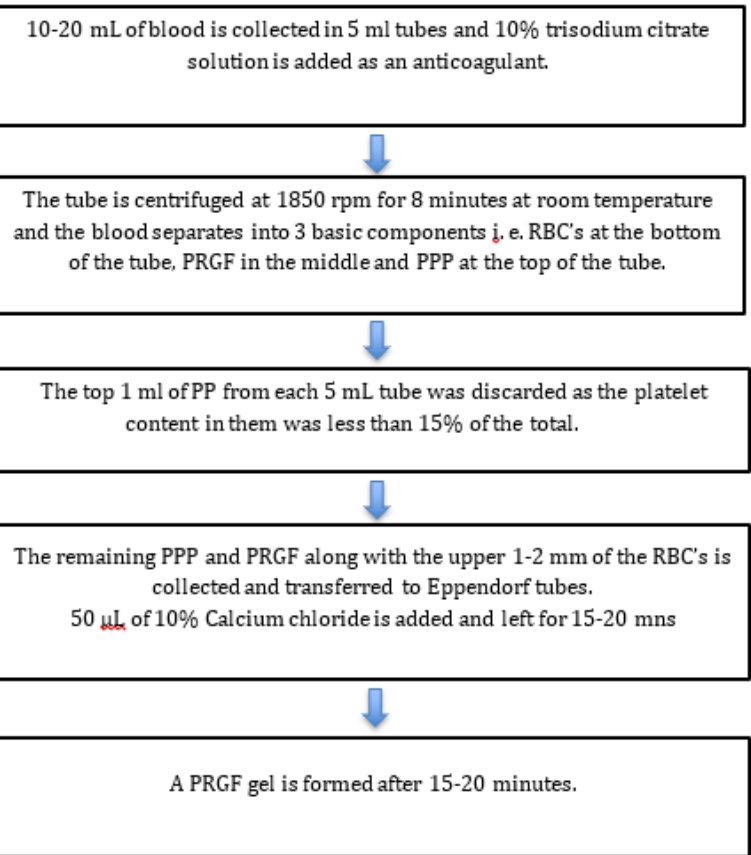

Figure 2: Steps in preparation of PRGF.

\section{Applications}

Studies of the application of PRGF in orthodontics and accelerated orthodontics are scarce. However, PRGF has been used in the other fields of dentistry for their regenerative properties such as [37].

- Improving osseointegration by soaking the implant in PRGF before placement.

- Support bone regeneration by via chemotactic and mitogenic effects on preosteoblastic and osteoblastic cells

\section{Drawbacks of PRGF [15]}

A high concentration of thrombin added during the preparation of PRGF allows thickening of the polymers of fibrin resulting in formation of rigid meshwork favoring the sealing of the biological tissues but impairing cytokine release and cellular migration.

\section{Platelet rich fibrin}

Platelet-rich fibrin (PRF) first described by Choukroun., et al. [38] is essentially a platelet concentrate containing platelets and growth factors in the form of fibrin membranes. It is prepared from autologous blood and is free of any anticoagulant and other artificial biochemical modifications.

PRF presents itself with several advantages compared to PRP and PRGF [15,26,39]:

- $\quad$ Easier preparation without any chemical manipulation of the blood making it an autologous preparation

- The clot produced forms a flexible matrix favoring the entrapment of cytokines and cells

- Its preparation is a simplified technique with minimum blood manipulation.

- No addition of any external agent such as thrombin is required.

- It comprises of a natural fibrin framework composed of growth factors that have activity for a relatively longer period.

- It is an economical and quick option compared to recombinant growth factors.

\section{Composition}

PRF is essentially an autologous leukocyte-platelet-rich fibrin matrix. It is a tetra molecular structure containing platelets, cytokine and stem cells that act as a biodegradable scaffold $[17,36]$.

PRF forms a natural blood clot with a dense fibrin network. This fibrin network contains cytokines, leukocytes, structural glycoproteins and growth factors such as platelet-derived growth factor, vascular endothelial growth factor, transforming growth factor b1, and glycoproteins such as thrombospondin-1 [37]. 
As the preparation of PRF does not involve the addition of any anticoagulant, as soon as the blood comes in contact with the glass surface of the tube, it begins to coagulate. The contact with a silica surface is required to activate the clot polymerization process. In addition, the silica particles unlike the bovine thrombin used for PRP preparation, are not cytotoxic [26].

\section{Method of preparation [37]}

The method of preparation involves centrifugation of blood without addition of any anticoagulant or bovine thrombin. The blood sample is drawn and immediately centrifuged to obtain the product (Figure 3).

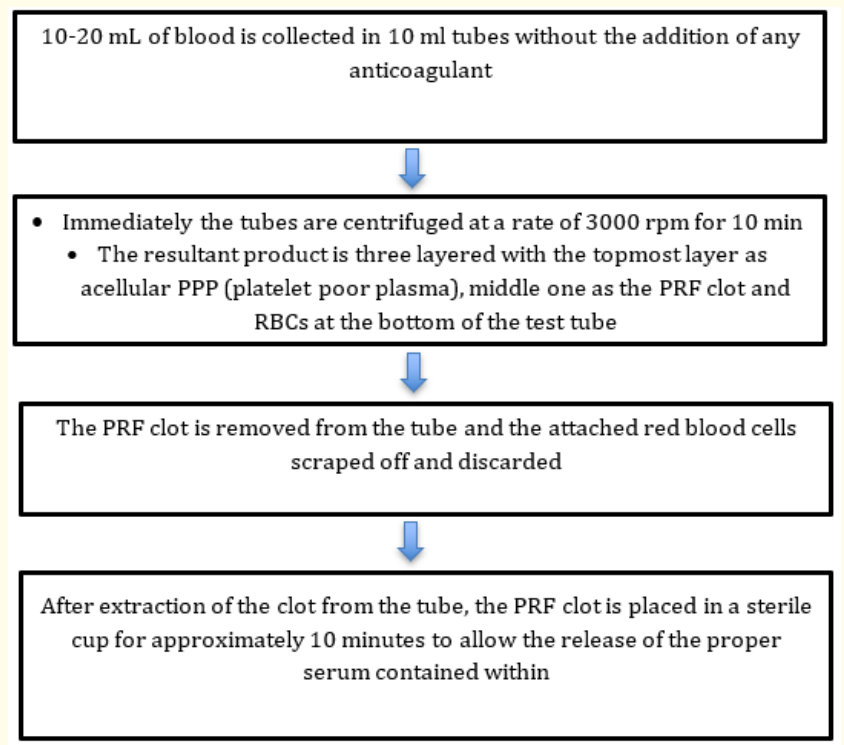

Figure 3: Steps in preparation of PRF.

\section{Applications}

\section{Accelerated orthodontics}

Tehranchi., et al. [39] conducted a human pilot study evaluating the effect of PRF (placed in extraction sockets) on orthodontic tooth movement (OTM). They found a possible positive efficacy of PRF application in the extraction socket for acceleration of OTM. However, there exists a paucity of conclusive human studies to ascertain the same results.

- PRF aids in achieving a reduction in probing depth in periodontal defects along with acting as an adjunct for palatal wound healing after harvesting a soft tissue graft [40].

- PRF can act as a potential scaffold in pulp revascularization procedures of necrotic immature permanent tooth as it is rich in growth factors [41].

- It can be used for preservation of alveolar ridge height and bone regeneration around immediate implants [42].

Drawbacks [43]

- The success of the PRF depends on the handling during the blood collection time and its transference for further process.

- Need of using a glass-coated tube for clot polymerization.

\begin{tabular}{|c|c|c|c|}
\hline S No & Growth factor & Source & Biologic action \\
\hline 1 & $\begin{array}{c}\text { Platelet-derived growth fac- } \\
\text { tor (PDGF) [4] }\end{array}$ & $\begin{array}{c}\text { Alpha granules (Platelets), } \\
\text { Macrophages, Endothelial cells, } \\
\text { Monocytes, fibroblasts }\end{array}$ & $\begin{array}{c}\text { Favor angiogenesis and collagen synthesis } \\
\text { Increase the rate of proliferation of stem cells. }\end{array}$ \\
\hline 2 & $\begin{array}{c}\text { Transforming Growth Factor- } \\
\text { B. [45] }\end{array}$ & $\begin{array}{c}\text { Platelets, T lymphocytes, neutro- } \\
\text { phils and bone extracts } \\
\text { cells to the place of repair activating fibroblasts, } \\
\text { osteoblasts, and chondroblasts proliferation }\end{array}$ \\
\hline 3 & $\begin{array}{c}\text { EGF (Epidermal Growth Fac- } \\
\text { tor) [46] }\end{array}$ & Epithelial cells & Inhibitory effect on osteoclasts \\
\hline 4 & $\begin{array}{c}\text { IGF 1 - (Insulin like growth } \\
\text { factor) [47,48] }\end{array}$ & $\begin{array}{c}\text { Osteoblasts, macrophages, } \\
\text { monocytes, chondrocytes }\end{array}$ & $\begin{array}{c}\text { Promotes chemotaxis and mitogenesis of epithe- } \\
\text { lial, mesenchymal cells and fibroblasts also induc- } \\
\text { ing tissue regeneration. }\end{array}$ \\
\hline 5 & $\begin{array}{c}\text { VEGF (Vascular endothelial } \\
\text { growth factor) [49] }\end{array}$ & $\begin{array}{c}\text { Macrophages, Platelets, Kerati- } \\
\text { nocytes }\end{array}$ & $\begin{array}{c}\text { Mediates growth, differentiation, and cellular } \\
\text { transforming and stimulates osteoblasts. } \\
\text { It is also involved in keratinocytes migration and } \\
\text { wound healing }\end{array}$ \\
\hline 6 & $\begin{array}{c}\text { HGF (Hepatocyte growth fac- } \\
\text { tor) [50] }\end{array}$ & Macrophages, Fibroblasts & $\begin{array}{c}\text { Signaling protein stimulating chemotaxis and } \\
\text { endothelial cell proliferation with specificity for } \\
\text { the vascular endothelial cells }\end{array}$ \\
\hline
\end{tabular}

Table 1: Growth factors released during wound healing and their biological actions. 


\begin{tabular}{|c|c|c|c|}
\hline Platelet Concentrate & PRP & PRGF & PRF \\
\hline Generation & $1^{\text {st }}$ & $1^{\text {st }}$ & $2^{\text {nd }}$ \\
\hline Coagulation product added & $\begin{array}{l}\text { Citrate phosphate dextrose adenine } \\
\text { followed by thrombin and calcium } \\
\text { chloride }\end{array}$ & $\begin{array}{l}\text { Sodium citrate and Calcium } \\
\text { chloride }\end{array}$ & None \\
\hline Speed of centrifugation & Slow & Very slow & Fast \\
\hline Amount obtainable & Moderate & Poor & Good \\
\hline Cost & High & High & Low \\
\hline Fibrin formation & None & Yes & Yes \\
\hline Fibrin morphology & & Tetramolecular & Tri molecular \\
\hline Leukocytes & & Nil & $65 \%$ \\
\hline Growth factors & $\checkmark$ & $\checkmark \checkmark$ & $\sqrt{ } \sqrt{ }$ \\
\hline Presentation form & Liquid/ Gel & $\begin{array}{c}\text { Liquid } \\
\text { Clot } \\
\text { Supernatant } \\
\text { Fibrin membrane }\end{array}$ & $\begin{array}{c}\text { Plugs } \\
\text { Exudate } \\
\text { Fibrin membrane }\end{array}$ \\
\hline
\end{tabular}

Table 2: Comparison between the first and second-generation platelet concentrates $[19,38]$.

\section{Conclusion}

Several in vitro and in vivo studies in the past have evaluated the efficiency of platelet concentrates and have established safe results for their use in humans. As a result, a wide of clinical applications have been established in dentistry where the concentrates are highly beneficial.

However, in the field of orthodontics there still exists a huge lacuna of the role of these products for accelerating tooth movement, as there is a paucity of any conclusive human studies concerning the same.

\section{Funding}

Not applicable.

\section{Conflict of Interest}

The authors declare that they have no conflict of interest.

\section{Availability of Data and Materials}

Not applicable.

\section{Bibliography}

1. Wise GE and King GJ. "Mechanisms of tooth eruption and orthodontic tooth movement". Journal of Dental Research 87.5 (2008): 414-434.
2. Bruno MB., et al. "A double-blind, randomized clinical trial assessing the effects of a single dose of preemptive anti-inflammatory treatment in orthodontic pain". Progress in Orthodontics 12.1 (2011): 2-7.

3. Yamaguchi M. "RANK/RANKL/OPG during orthodontic tooth movement". Orthodontics and Craniofacial Research 12.2 (2009): 113-119.

4. Agrawal M and Agrawal V. "Platelet rich fibrin and its applications in dentistry-A review article". National Journal of Medical and Dental Research 2.3 (2014): 51.

5. Frost HM. "The regional acceleratory phenomenon: a review". Henry Ford Hospital Medical Journal 31.1 (1983): 3-9.

6. Mangal U. "Influence of platelet rich plasma on orthodontic tooth movement: a review". Biomedical and Pharmacology Journal 10.3 (2017): 1463-1468.

7. Uzuner FD and Darendeliler N. "Dentoalveolar surgery techniques combined with orthodontic treatment: A literature review". European Journal of Dentistry 7.2 (2013): 257-265.

8. Kale S., et al. "Comparison of the effects of 1,25 dihydroxycholecalciferol and prostaglandin E2 on orthodontic tooth movement". American Journal of Orthodontics and Dentofacial Orthopedics 125.5 (2004): 607-614. 
9. Seifi M., et al. "The effect of prostaglandin E2 and calcium gluconate on orthodontic tooth movement and root resorption in rats". European Journal of Orthodontics 25.2 (2003): 199-204.

10. Collins MK and Sinclair PM. "The local use of vitamin D to increase the rate of orthodontic tooth movement". American Journal of Orthodontics and Dentofacial Orthopedics 94.4 (1988): 278-284.

11. Kobayashi Y., et al. "Effects of local administration of osteocalcin on experimental tooth movement". Angle of Orthodontics 68.3 (1998): 259-266.

12. Doshi-Mehta G., et al. "Efficacy of low-intensity laser therapy in reducing treatment time and orthodontic pain: a clinical investigation". American Journal of Orthodontics and Dentofacial Orthopedics 141.3 (2012): 289-297.

13. Ganesh G., et al. "Effect of low level laser therapy on rate of orthodontic tooth movement". European Journal of Pharmaceutical and Medical Sciences 5.10 (2008): 274-277.

14. Davidovitch Z., et al. "Electric currents, bone remodeling, and orthodontic tooth movement. I. The effect of electric currents on periodontal cyclic nucleotides". American Journal of Orthodontics 77.1 (1980): 14-32.

15. Giannini S., et al. "Comparison between PRP, PRGF and PRF: lights and shadows in three similar but different protocols". European Review for Medical and Pharmacological Sciences 19.6 (2015): 927-930.

16. Gupta SJ., et al. "Efficacy of platelet-rich fibrin vs. enamel matrix derivative in the treatment of periodontal intrabony defects: a clinical and cone beam computed tomography study". Journal of the International Academy of Periodontology 16.3 (2014): 86-96.

17. Anitua E., et al. "Fibroblastic response to treatment with different preparations rich in growth factors". Cell Proliferation 42.2 (2009): 162-170.

18. Caruana A., et al. "From Platelet-Rich Plasma to Advanced Platelet-Rich Fibrin: Biological Achievements and Clinical Advances in Modern Surgery". European Journal of Dentistry 13.2 (2019): 280-286.

19. Dohan DM., et al. "Platelet-rich fibrin (PRF): a second-generation platelet concentrate. Part II: platelet-related biologic features". Oral Surgery, Oral Medicine, Oral Pathology, Oral Radiology, and Endodontology 101.3 (2006): e45-e50.
20. Sacco L. "Lecture, International academy of implant prosthesis and osteoconnection" 12 (2016): 4.

21. Choukroun J. "Advanced PRF, \& i-PRF: platelet concentrates or blood concentrates". Journal of Periodontal Medicine and Clinical Practice 1.1 (2014): 3.

22. Mourão CF, et al. "Obtention of injectable platelets rich-fibrin (i-PRF) and its polymerization with bone graft: technical note". Revista do Colégio Brasileiro de Cirurgiões 42.6 (2015): 421-423.

23. Marx RE., et al. "Platelet-rich plasma: Growth factor enhancement for bone grafts". Oral Surgery, Oral Medicine, Oral Pathology, Oral Radiology, and Endodontology 85.6 (1998): 638-646.

24. Marx RE. "Platelet-rich plasma (PRP): what is PRP and what is not PRP?". Implant Dentistry 10.4 (2001): 225-228.

25. Weibrich G., et al. "Effect of platelet concentration in plateletrich plasma on peri-implant bone regeneration". Bone 34.4 (2004): 665-671.

26. Malandkar A., et al. "Platelet Rich plasma in Orthodontics - A review". International Journal of Innovative Science and Research Technology 4.8 (2016): 657-662.

27. Sunitha Raja V., et al. "Platelet-rich fibrin: evolution of a second-generation platelet concentrate". Indian Journal of Dental Research 19. (2008): 42-46.

28. Sonnleitner D., et al. "A simplified technique for producing platelet-rich plasma and platelet concentrate for intraoral bone grafting techniques: a technical note". International Journal of Oral and Maxillofacial Implants 15.6 (2000): 879-882.

29. Graziani F., et al. "The in vitro effect of different PRP concentrations on osteoblasts and fibroblasts". Clinical Oral Implants Research 17.2 (2006): 212-219.

30. Rashid A., et al. "Effect of platelet-rich plasma on orthodontic tooth movement in dogs". Orthodontics and Craniofacial Research 20.2 (2017): 102-110.

31. Güleç A., et al. "Effects of local platelet-rich plasma injection on the rate of orthodontic tooth movement in a rat model: A histomorphometric study". American Journal of Orthodontics and Dentofacial Orthopedics 151.1 (2017): 92-104.

32. Gupta C., et al. "Alveolar bone graft with Platelet Rich Plasma in cleft alveolus". Journal of Oral Biology and Craniofacial Research 3.1 (2013): 3-8. 
33. Lee C., et al. "A quantitative radiological assessment of outcomes of autogenous bone graft combined with platelet-rich plasma in the alveolar cleft". International Journal of Oral and Maxillofacial Surgery 38.2 (2009): 117-125.

34. Kaur P and Maria A. "Efficacy of platelet rich plasma and hydroxyapatite crystals in bone regeneration after surgical removal of mandibular third molars". Journal of Oral and Maxillofacial Surgery 12.1 (2013): 51-59.

35. Kawase T. "Platelet-rich plasma and its derivatives as promising bioactive materials for regenerative medicine: basic principles and concepts underlying recent advances". Odontology 103.2 (2015): 126-135.

36. Anitua E. "The use of plasma-rich growth factors (PRGF) in oral surgery". Practical Procedures and Aesthetic Dentistry 13.6 (2001): 487-493.

37. Boras VV., et al. "Plasma rich in growth factors in dentistry". Australasian Medical Journal 10.6 (2017): 497.

38. Choukroun J., et al. "PRF: an opportunity in perio-implantology”. Implantodontie 42 (2000): 55-62.

39. Tehranchi A., et al. "The effect of autologous leukocyte platelet rich fibrin on the rate of orthodontic tooth movement: A prospective randomized clinical trial". European Journal of Dentistry 12.3 (2018): 350.

40. Chang YC and Zhao JH. "Effects of platelet-rich fibrin on human periodontal ligament fibroblasts and application for periodontal infrabony defects". Australian Dental Journal 56.4 (2011): 365-371.

41. Keswani D and Pandey RK. "Revascularization of an immature tooth with a necrotic pulp using platelet-rich fibrin: a case report". International Endodontic Journal 46.11 (2013): 10961104.

42. Simonpieri A., et al. "Current knowledge and perspectives for the use of platelet-rich plasma (PRP) and platelet-rich fibrin (PRF) in oral and maxillofacial surgery part 2: Bone graft, implant and reconstructive surgery". Current Pharmaceutical Biotechnology 13.7 (2012): 1231-1256.

43. Borie E., et al. "Platelet-rich fibrin application in dentistry: a literature review". International Journal of Clinical and Experimental Medicine 8.5 (2015): 7922-7929.

44. Anitua E. "Plasma rich in growth factors: preliminary results of use in the preparation of future sites for implants". International Journal of Oral and Maxillofacial Implants 14.4 (1998): 529-535.
45. Miyazono K. "Positive and negative regulation of TGF-beta signaling". Journal of Cell Science 113 (2000): 1101-1109.

46. Consolaro A., et al. "Saucerization of osseointegrated implants and planning of simultaneous orthodontic clinical cases". Dental Press Journal of Orthodontics 15 (2010): 19-30.

47. Kurten RC., et al. "Coordinating epidermal growth factor-induced motility promotes efficient wound closure". American Journal of Physiology-Cell Physiology 288.1 (2005): C109-C121.

48. E Govoni K. "Insulin-like growth factor-I molecular pathways in osteoblasts: potential targets for pharmacological manipulation". Current Molecular Pharmacology 5.2 (2012): 143-152.

49. Ferrara N and Gerber HP. "The role of vascular endothelial growth factor in angiogenesis". Acta haematologica 106.4 (2001): 148-156.

50. Anitua E., et al. "Platelet-rich plasma: preparation and formulation". Operative Techniques in Orthopaedics 22.1 (2012): 2532.

\section{Assets from publication with us}

- Prompt Acknowledgement after receiving the article

- Thorough Double blinded peer review

- Rapid Publication

- Issue of Publication Certificate

- High visibility of your Published work

Website: www.actascientific.com/

Submit Article: www.actascientific.com/submission.php

Email us: editor@actascientific.com

Contact us: +919182824667 